\title{
Connecting Scrambling and Work Statistics for Short-Range Interactions in the Harmonic Oscillator
}

\author{
M. Mikkelsen $\odot,{ }^{1,2, *}$ T. Fogarty $\odot,{ }^{1}$ and Th. Busch $\oplus^{1}$ \\ ${ }^{1}$ Quantum Systems Unit, OIST Graduate University, Onna, Okinawa 904-0495, Japan \\ ${ }^{2}$ Department of Physics, Kindai University, Higashi-Osaka City, Osaka 577-8502, Japan
}

(Received 6 October 2020; accepted 21 January 2022; published 18 February 2022)

\begin{abstract}
We investigate the relationship between information scrambling and work statistics after a quench for the paradigmatic example of short-range interacting particles in a one-dimensional harmonic trap, considering up to five particles numerically. In particular, we find that scrambling requires finite interactions, in the presence of which the long-time average of the squared commutator for the individual canonical operators is directly proportional to the variance of the work probability distribution. In addition to the numerical results, we outline the mathematical structure of the $N$-body system which leads to this outcome. We thereby establish a connection between the scrambling properties and the induced work fluctuations, with the latter being an experimental observable that is directly accessible in modern cold-atom experiments.
\end{abstract}

DOI: 10.1103/PhysRevLett.128.070605

The response to a sudden change in the Hamiltonian is a topic which has led to many valuable insights into the physics of quantum many-particle systems in recent years. Quenches have been used to probe phase transitions [1-7], explore the orthogonality catastrophe [8-13], and investigate irreversibility, thermodynamics, and equilibration properties [14-18]. For example, systems that obey the eigenstate thermalization hypothesis have been shown to thermalize $[15,16]$ while integrable systems do not $[14,17]$. Studying the dynamical response of a system to a sudden quench usually entails calculating the time evolution of expectation values of observables such as the momentum distribution. However, one can also characterize a quench more broadly, for example through operator-independent (aside from the final Hamiltonian $\hat{H}_{F}$ ) quantities such as the diagonal ensemble [15,19] and the closely related experimentally measurable work probability distribution [18,20-24]. The statistical moments of the work probability distribution, $\left\langle W^{\alpha}\right\rangle=\operatorname{Tr}\left[\left(\hat{H}_{F}-\hat{H}_{I}\right)^{\alpha} \hat{\rho}_{I}\right]$, where $\hat{H}_{I}$ is the initial Hamiltonian and $\hat{\rho}_{I}$ is the initial state, are often used to give an indication of the irreversibility of the quench process [21]. One example of this is the irreversible work $\left\langle W_{\text {irr }}\right\rangle=\langle W\rangle-\Delta F$ which quantifies the disparity between the average work and the free energy during a nonquasistatic process. Further insight can be gained through the variance of nonequilibrium fluctuations about the average, $\Delta W^{2}=\left\langle W^{2}\right\rangle-\langle W\rangle^{2}$, which is of interest in the field of

Published by the American Physical Society under the terms of the Creative Commons Attribution 4.0 International license. Further distribution of this work must maintain attribution to the author(s) and the published article's title, journal citation, and DOI. statistical quantum thermodynamics [16,20,25] and has been suggested as a probe of critical behavior [26,27].

Since the work probability distribution is related to the delocalization of the initial state in the Hilbert space defined by the eigenstates of the final Hamiltonian, it is natural to characterize this further by investigating the delocalization dynamics. This process is often referred to as scrambling $[28,29]$, whereby over time the initial state can no longer be reconstructed from local measurements alone. One particular measure of this scrambling is the expectation value of the squared commutator of two operators $\hat{A}(t)=e^{i \hat{H} t} \hat{A} e^{-i \hat{H} t}$ and $\hat{B}, C_{A B}(t)=\left\langle[\hat{A}(t), \hat{B}]^{2}\right\rangle$ [28], which can be rewritten in terms of time-dependent correlation functions as $C_{A B}(t)=D_{A B}(t)+I_{A B}(t)-2 \operatorname{Re}\left[F_{A B}(t)\right]$, with

$$
\begin{aligned}
& D_{A B}(t)=\left\langle\hat{B}^{\dagger} \hat{A}^{\dagger}(t) \hat{A}(t) \hat{B}\right\rangle, \\
& I_{A B}(t)=\left\langle\hat{A}^{\dagger}(t) \hat{B}^{\dagger} \hat{B} \hat{A}(t)\right\rangle, \\
& F_{A B}(t)=\left\langle\hat{A}^{\dagger}(t) \hat{B}^{\dagger} \hat{A}(t) \hat{B}\right\rangle .
\end{aligned}
$$

Most work in recent years has focused on the four-point out-of-time ordered correlation (4-OTOC) function $F_{A B}(t)$, as $D_{A B}(t)$ is time ordered and $I_{A B}(t)=\left\langle\hat{A}^{\dagger} \hat{B}^{\dagger}(-t) \hat{B}(-t) \hat{A}\right\rangle$ is anti-time-ordered for an eigenstate of the Hamiltonian. The squared commutator and the 4-OTOC were initially proposed as measures of quantum chaos [30] but have recently been shown to be powerful tools for studying information scrambling in nonchaotic systems as well, for example near quantum critical points [31-33], in the presence of many-body entanglement and coherence $[34,35]$, and in quantum thermodynamics [36,37]. For 
initial states that are not eigenstates, e.g., states after a quench, $I_{A B}(t)$ is also not time ordered and called a threepoint OTOC (3-OTOC) [38]. One can see that $I_{A B}(t)$ is readily interpretable as a time-reversal test, i.e., it corresponds to taking the expectation value of $\hat{B}^{\dagger} \hat{B}$ with the quantum-state $\hat{A}(t)|\psi\rangle$. It therefore measures how much the time-reversal symmetry is broken by the application of the operator $\hat{A}$.

While in discrete systems schemes for measuring the OTOCs have been experimentally implemented using a time-reversal protocol [34], in continuum systems such a direct implementation is extremely difficult as it requires reversing the kinetic energy terms. Therefore, finding a connection between information scrambling and other measures of irreversibility, particularly ones that can be measured in continuum systems is important. While progress toward such an understanding has recently been made [36,38-41], we will focus in this work on nonchaotic systems and look at experimentally available cold-atom systems of interacting bosons in quasi-one-dimensional traps. Such systems offer an ideal test bed to study nonequilibrium dynamics as advances in the experimental manipulation of single- and few-body systems allows for precise control over their interactions and trapping potentials $[42,43]$. The total number of particles can also be tuned deterministically allowing one to explore the crossover between few- and many-body physics [44]. They are therefore highly suitable to consider how information scrambling emerges after sudden quenches, specifically as a function of finite interactions between the particles.

The system we consider consists of $N$ particles and can be described by the dimensionless Hamiltonian

$\hat{H}=\sum_{j=1}^{N}\left[-\frac{1}{2} \frac{\partial^{2}}{\partial \hat{x}_{j}^{2}}+\frac{1}{2} \Omega^{2}(t) \hat{x}_{j}^{2}\right]+\sum_{k>j} g \delta\left(\hat{x}_{k}-\hat{x}_{j}\right)$,

where the interactions and trap frequency are parametrized by $g$ and $\Omega(t)$, respectively. To explore nonequilibrium scrambling in this system we consider the canonical operators $\hat{x}_{j}$ and $\hat{p}_{j}$ after a sudden change of the trapping potential described by $\Omega(t)=\gamma+\Theta(t)(1-\gamma)$, where $\Theta(t)$ is the Heaviside step function. The trap strength in the initial Hamiltonian is therefore given by $\gamma$, while the final Hamiltonian has a trap strength of unity. This allows us to scale all relevant quantities in units of the final Hamiltonian and all results only depend on $\gamma$, which then quantifies the strength of the quench and whether the trap is compressed $(\gamma<1)$ or expanded $(\gamma>1)$. We keep the interaction strength fixed throughout the dynamics with $g>0$ describing repulsive interactions. This allows us to clearly identify the effects of finite interactions on the information scrambling and work statistics after the quench of the trapping potential.
Sudden quenches are characterized by the eigenspace of the final Hamiltonian $\hat{H}_{F}\left|\psi_{j}\right\rangle=E_{j}\left|\psi_{j}\right\rangle$ and the overlap coefficients $c_{j}=\left\langle\psi_{j} \mid \psi^{I}\right\rangle$, where $\left|\psi^{I}\right\rangle$ is the initial state with energy $E^{I}$. This allows one to write the contributions to the squared commutator as

$$
\begin{gathered}
D_{A B}(t)=\sum_{j, k, n, m} c_{j}^{*} c_{k} e^{-i\left(E_{m n}\right) t} B_{j n}^{\dagger}\left\langle\hat{A}^{\dagger} \hat{A}\right\rangle_{n m} B_{m k}, \\
I_{A B}(t)=\sum_{j, k, n, m} c_{j}^{*} c_{k} e^{-i\left(E_{k j}+E_{n m}\right) t} A_{j n}^{\dagger}\left\langle\hat{B}^{\dagger} \hat{B}\right\rangle_{n m} A_{m k}, \\
F_{A B}(t)=\sum_{j, k, n, m} c_{j}^{*} b_{k} e^{-i\left(E_{k j}+E_{n m}\right) t} A_{j n}^{\dagger} B_{n m}^{\dagger} A_{m k},
\end{gathered}
$$

where $\quad b_{j}=\left\langle\psi_{j}|\hat{B}| \psi^{I}\right\rangle, \quad A_{j k}=\left\langle\psi_{j}|\hat{A}| \psi_{k}\right\rangle, \quad\left\langle\hat{A}^{\dagger} \hat{A}\right\rangle_{n m}=$ $\left\langle\psi_{n}\left|\hat{A}^{\dagger} \hat{A}\right| \psi_{m}\right\rangle$, and the other operator matrix elements are defined similarly. The energy differences are given by $E_{m n}=E_{m}-E_{n}$. The statistical moments of the work probability distribution can be expressed as $\left\langle W^{\alpha}\right\rangle=$ $\sum_{j}\left|c_{j}\right|^{2}\left(E_{j}-E^{I}\right)^{\alpha}$ with $\alpha=1,2, \ldots$ [21]. The variance $\Delta W^{2}=\left\langle W^{2}\right\rangle-\langle W\rangle^{2}$ will be used as a quantifier of the irreversibility of the quench dynamics, while the information scrambling will be gauged by the infinite time average of the squared commutator $\bar{C}_{A B}=\lim _{T \rightarrow \infty}(1 / T) \times$ $\int_{0}^{T}\left\langle[\hat{A}(t), \hat{B}]^{2}\right\rangle d t$. Time-averaged behavior has recently attracted more attention and has been connected to the description of quantum phases [31,33].

The Hamiltonian in Eq. (4) possesses analytical manybody solutions in the noninteracting limit $g=0$ and the Tonks-Girardeau (TG) limit of infinite repulsive interactions, $g \rightarrow \infty$. In both cases the many-body system is described by a harmonic spectrum which elicits self-similar dynamics after changes to the trapping frequency $[45,46]$, and the scrambling of canonical operators in these limits therefore simply reflects the single-particle breathing mode following a trap quench. In fact, it can be shown that the time averaged scrambling in both limits for the individual canonical operators $\left[\hat{A}_{i}(t), \hat{B}_{j}\right]^{2}$, where $\hat{A}_{i}=\hat{x}_{i}, \hat{p}_{i}$ and $\hat{B}_{j}=\hat{x}_{j}, \hat{p}_{j}$ is given by [47] $\bar{C}_{A_{j}, B_{k}}^{g=0}=\bar{C}_{A_{j}, B_{k}}^{g \rightarrow \infty}=\frac{1}{2} \delta_{j k}$. The scrambling is therefore independent of both the system-size and the strength of the trap quench $\gamma$.

For finite interactions, $g>0$, the energy levels acquire nontrivial shifts $E_{j}^{g>0}=E_{j}^{g=0}+\Delta_{j}$ which destroy the regularity of the harmonic oscillator spectrum. This leads to complex dynamics which do not admit a single particle description and introduces correlations between the particles. While large systems become computationally intractable, few-body systems are solvable while retaining the physics stemming from the finite contact interactions [51]. For $N=2$ particles analytic solutions exist $[52,53]$ which can be used to find an analytic expression for the full squared commutator [47]. For larger systems, $N=3,4,5$, 
one must solve the Hamiltonian in Eq. (4) numerically which we do by utilizing exact diagonalization techniques [54] with an effective interaction approach [55] and an optimized choice of the many-body basis [56]. We will focus on the dynamics of $\left[\hat{x}_{1}(t), \hat{x}_{1}\right]^{2}$, as other combinations of canonical operators give similar results [47].

In Figs. 1(a) and 1(b) the variance of the work distribution and average scrambling is shown as a function of the quench strength $\gamma$ for finite interactions $g=5$ and different system sizes. Reducing $\gamma$ (increasing the compression of the trap) drives the system further from equilibrium and therefore increases both the variance and the information scrambling. For the different system sizes the variance and the average scrambling are rescaled by $N^{b_{W}}$ and $N^{b_{C}}$, respectively, where the exponents $b_{W}$ and $b_{C}$ are found by extrapolating the behavior of the system in the analytically solvable limits $g=\{0, \infty\}$. In these the variance as a function of $N$ and $\gamma$ is $\Delta W_{g=0}^{2}=(N / 8)[\gamma-(1 / \gamma)]^{2}$ and $\Delta W_{g=\infty}^{2}=\left[N\left(N^{2}+2\right) / 24\right][\gamma-(1 / \gamma)]^{2}$, which evinces that the interaction only affects how the system size scales. For finite interactions we therefore fit the function $\Delta W_{g, N}^{2}=N^{b_{W}(g)} \lambda_{W}(g, N)[\gamma-(1 / \gamma)]^{2}$ with the exponent having values $1<b_{W}(g)<3$ which are $g$ dependent. Similarly for the time-averaged squared commutator the following function gives a good fit $\bar{C}_{x_{1}, x_{1}}=$ $N^{b_{C}(g)} \lambda_{C}(g, N)\left\{[\gamma-(1 / \gamma)]^{2}+k_{C}(g, N)\right\}$. For up to $N=$ 5 particles the leading exponents of the system size are found to be $b_{W}(5) \approx 2$ and $b_{C}(5) \approx 1.7$.
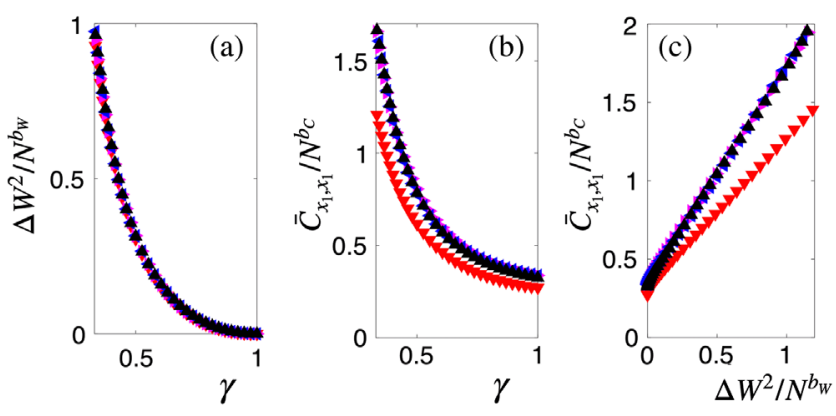

(d)

\begin{tabular}{|c|c|c|c|c|}
\hline$N$ & 2 & 3 & 4 & 5 \\
\hline$\lambda_{W}$ & 0.13 & 0.13 & 0.14 & 0.14 \\
\hline$\lambda_{C}$ & 0.12 & 0.18 & 0.19 & 0.18 \\
\hline$k_{C}$ & 2.6 & 2.0 & 2.0 & 2.0 \\
\hline
\end{tabular}

FIG. 1. (a) Variance and (b) time-averaged squared commutator as a function of the quench strength $\gamma$ for $g=5$. (c) Timeaveraged squared commutator as a function of the variance. The colors correspond to $N=2$ (red), $N=3$ (magenta), $N=4$ (blue), and $N=5$ (black). The time-averaged squared commutator is scaled with $N^{b_{C}}$, while the variance is scaled with $N^{b_{W}}$, with $b_{C}(5)=1.7$ and $b_{W}(5)=2$. (d) Corresponding fitting components as a function of particle number.
In Fig. 1(d) we list the numerically obtained values of the remaining fitting constants showing that they quickly converge for $N \geq 3$, which can also be seen in Figs. 1(a) and 1(b) as the data for $N=3,4,5$ show strong convergence. In Fig. 1(c) we plot $\bar{C}_{x_{1}, x_{1}}$ as a function of the variance $\Delta W^{2}$, showing that the average information scrambling is linearly proportional to the work fluctuations. For a system with finite interactions the information scrambling is therefore closely related to the irreversible nonequilibrium excitations created by the trap quench, something which is absent in the $g=\{0, \infty\}$ limits where $\bar{C}_{x_{1}, x_{1}}=1 / 2$ and therefore does not depend on the system size $N$ or quench strength $\gamma$.

The results in Fig. 1 can be explained in more detail by considering the structure of the Hamiltonian and the squared commutator analytically. To do this, we first outline some generic conditions under which the squared commutator will simplify and which are applicable to other similar models. First, we consider a nondegenerate system [condition (i)], which is the case in many situations of interest. From Eqs. (5)-(7) one can see that contributions to the infinite-time average of the squared commutator are only obtained when the complex exponential equals 1 , which means that $D_{A B}(t)$ has contributions whenever $E_{m}=E_{n}$. The contributions to $I_{A B}$ and $F_{A B}$ can be split into three cases: the energy differences can be pairwise zero in the case where $E_{k}=E_{j}$ and $E_{m}=E_{n}$ or the sum can be zero when $E_{k}=E_{m}$ and $E_{n}=E_{j}$. Finally, it is also possible that $E_{k}-E_{j}+E_{n}-E_{m}=0$ for $j \neq k \neq n \neq m$. However, we will consider an additional constraint on the spectrum, namely $E_{k}-E_{j}+E_{n}-E_{m} \neq 0$ for $j \neq k \neq n \neq m$ [condition (ii)] which ensures that these terms have no contributions. This often holds in chaotic systems [57], although it needs to be explicitly shown for any system of interest. The resulting time averages can therefore be written as

$$
\begin{gathered}
\bar{D}_{A B}=\sum_{j, k, n} c_{j}^{*} c_{k} B_{j n}^{\dagger}\left\langle\hat{A}^{\dagger} \hat{A}\right\rangle_{n n} B_{n k} \\
\bar{I}_{A B}=\sum_{j, n}\left|c_{j}\right|^{2} A_{j n}^{\dagger}\left\langle\hat{B}^{\dagger} \hat{B}\right\rangle_{n n} A_{n j}+\sum_{j \neq k} c_{j}^{*} c_{k} A_{j j}^{\dagger}\left\langle\hat{B}^{\dagger} \hat{B}\right\rangle_{j k} A_{k k}, \\
\bar{F}_{A B}=\sum_{j, n} c_{j}^{*} b_{j} A_{j n}^{\dagger} B_{n n}^{\dagger} A_{n j}+\sum_{j \neq k} c_{j}^{*} b_{k} A_{j j}^{\dagger} B_{j k}^{\dagger} A_{k k} .
\end{gathered}
$$

If the system obeys a final constraint on the matrix elements of the operators with respect to the eigenbasis of the final Hamiltonian, namely $B_{k k}=A_{k k}=0$ [condition (iii)] the second term in Eq. (9) and both terms in Eq. (10) will be zero. This constraint is much less generic than those on the spectrum and is only fulfilled by certain classes of models and operators. In general, it will be obeyed by 
systems with an odd-even parity symmetry and for operators which change the parity of a state.

The time averages for any system which fulfills conditions (i)-(iii) reduce to $\bar{F}_{A B}=0$ and $\bar{C}_{A B}=\bar{D}_{A B}+\bar{I}_{A B}$, with

$$
\bar{D}_{A B}=\sum_{j, k} c_{j} c_{k} K_{j k}^{B A}, \quad \bar{I}_{A B}=\sum_{j}\left|c_{j}\right|^{2} K_{j j}^{A B},
$$

where $K_{j k}^{A B}=\sum_{n} A_{j n}^{\dagger}\left\langle\hat{B}^{\dagger} \hat{B}\right\rangle_{n n} A_{n k} . \bar{I}_{A B}$ is given as the diagonal ensemble expectation value of an emergent operator and is therefore directly related to the work statistics of the quench with no dependence on the sign of the overlap coefficients. $\bar{D}_{A B}$ is given as a sum over all the off-diagonal values of a similar emergent operator which means that the sign of the overlap coefficients matter and negative and positive contributions can interfere destructively.

The symmetries of a system of $N$ interacting particles in a harmonic trap have been thoroughly explored [51,58-62], and the many-body Hamiltonian can be rewritten in terms of a center-of-mass $(\mathrm{CM})$ coordinate $R=(1 / \sqrt{N}) \times$ $\sum_{n=1}^{N} x_{n}$ and $N-1$ relative Jacobi coordinates (REL) given by $y_{n}=\sqrt{[n /(n+1)]}\left[(1 / n) \times \sum_{j=1}^{n} x_{j}-x_{n+1}\right]$. The system is then separable as $\hat{H}=\hat{H}_{\mathrm{CM}}+\hat{H}_{\mathrm{REL}}$, where the center-of-mass Hamiltonian corresponds to a single particle harmonic oscillator with frequency $\Omega(t)$, while the relative Hamiltonian contains the effects of interactions and is given by

$$
\begin{aligned}
\hat{H}_{\mathrm{REL}}= & \sum_{j=1}^{N-1}\left[-\frac{1}{2} \frac{\partial^{2}}{\partial \hat{y}_{j}^{2}}+\frac{1}{2} \Omega^{2}(t) \hat{y}_{j}^{2}\right] \\
& +\sum_{k>j} g \delta\left(\sqrt{\frac{j-1}{j}} \hat{y}_{j-1}-\sqrt{\frac{k-1}{k}} \hat{y}_{k-1}\right. \\
& \left.-\sum_{n=j}^{k} \frac{1}{\sqrt{n(n+1)}} \hat{y}_{n}\right) .
\end{aligned}
$$

Rewriting the lab-frame position operators as $\hat{x}_{n}=$ $(\hat{R} / \sqrt{N})+\hat{Y}_{n}$, where $\hat{Y}_{n}=\sum_{j=n}^{N-1}(1 / \sqrt{j(j+1)}) \hat{y}_{j}-$ $\sqrt{[(n-1) / n]} \hat{y}_{n-1}$ is the collective relative coordinate, allows us to recast the infinite time average as [47] $\bar{C}_{\hat{x}_{j}, \hat{x}_{k}}=\bar{C}_{\hat{Y}_{j}, \hat{Y}_{k}}+\left(1 / N^{2}\right) \bar{C}_{\hat{R}, \hat{R}}$. The infinite time average is therefore given simply as the sum of the CM and REL averages and this also holds when considering the momentum operators $\bar{C}_{\hat{x}_{j}, \hat{p}_{k}}$ and $\bar{C}_{\hat{p}_{j}, \hat{p}_{k}}$ [47].

For the CM coordinates the squared commutator is equivalent to the non-interacting system and given by $\bar{C}_{\hat{R}, \hat{R}}=\frac{1}{2}[47,63]$. This contribution to the full scrambling decreases with the system size as $\left(1 / 2 N^{2}\right)$. The average scrambling in the system after a quench is therefore entirely determined by the relative-coordinate sector for which state-dependent energy shifts for the even parity states resulting from the interaction ensure that condition (ii) is obeyed. As the Hamiltonian has a full reflection symmetry with respect to the Jacobi coordinates $\hat{y}_{n}[60]$ one can prove condition (iii) for the individual operators and therefore the squared commutator for $\hat{Y}_{n}$ fulfills conditions (i)-(iii) and has an infinite time average given by Eq. (11) [47].

In order to show that $\bar{C}_{\hat{x}_{1}, \hat{x}_{1}}$ is proportional to the work fluctuations, however, we also require knowledge of the emergent operator $K^{Y_{1}, Y_{1}}$. From the analytically solvable $N=2$ case we find that $K^{Y_{1}, Y_{1}}$ from Eq. (11) is approximately a tridiagonal matrix with the largest contribution from the elements $K_{j j}^{Y_{1}, Y_{1}}, K_{j, j+1}^{Y_{1}, Y_{1}}$, and $K_{j, j-1}^{Y_{1}, Y_{1}}$ which scale with leading terms proportional to $\left(E_{j}^{\mathrm{REL}}\right)^{2}$. The second moment of the work probability distribution is given as $\left\langle W_{\mathrm{REL}}^{2}\right\rangle=$ $\sum_{j}\left|c_{j}\right|^{2}\left(E_{j}^{\mathrm{REL}}-E^{I}\right)^{2}$, while $\bar{I}_{Y_{1}, Y_{1}} \propto \sum_{j}\left|c_{j}\right|^{2}\left(E_{j}^{\mathrm{REL}}\right)^{2}$ as it is only diagonal in $K_{j j}^{Y_{1}, Y_{1}}$. While this clearly links the dynamics of the correlation functions to the second moment of the work probability distribution, it also holds for its variance $\Delta W^{2}=\sum_{j}\left|c_{j}\right|^{2}\left(E_{j}^{\mathrm{REL}}\right)^{2}-\left(\sum_{j}\left|c_{j}\right|^{2} E_{j}^{\mathrm{REL}}\right)^{2}$ as $\left\langle W^{2}\right\rangle \propto\langle W\rangle^{2}$. A similar argument can be made for $\bar{D}_{Y_{1}, Y_{1}}$ which can effectively be described as a sum over the tridiagonal elements of $K^{Y_{1}, Y_{1}}$ [47]. This final condition (iv) $\left[K_{j j}^{A, B} \propto\left(E_{j}^{\mathrm{REL}}\right)^{2}\right]$ is required to link the scrambling to the work fluctuations. While this is satisfied for a harmonic trap one cannot expect this result to generalize to other systems. However, the scrambling in any system which obeys conditions (i)-(iii) will be closely connected to the work statistics through Eq. (11), although the relation can be more complicated depending on the properties of $K^{A, B}$.

As noted previously, there is no connection between the variance and the information scrambling when the particles are in the limits of zero and infinite interactions, however, for finite interactions a linear relationship was found. Next, we explore how this manifests as a function of the interaction strength for $N=2$. Using Eq. (11) the infinite-time average of the squared commutator $\bar{C}_{\hat{x}_{1}, \hat{x}_{1}}$ can be calculated as a function of the interaction $g$, which is shown in Fig. 2(a). The information scrambling increases with increasing interactions and reaches asymptotic values for $g \rightarrow 0$ and $g \rightarrow \infty$. However, these asymptotic values are different from the known values in the limits $g=\{0, \infty\}$, given as $\bar{C}_{\hat{x}_{1}, \hat{x}_{1}}=1 / 2$ (black triangles in the figure). In contrast, the work fluctuations show a smooth crossover to the limiting values (red lines and triangles, respectively).

The difference between the asymptotic values of the squared commutator and the limiting values at $g=\{0, \infty\}$ shows that the scrambling is very sensitive to small deviations from the harmonic oscillator spectrum on infinitely long timescales. To check this result we compute the full time-dependent OTOCs in Eqs. (5)-(7) and numerically find their time average in the range $t \in[0,200 \pi]$ 

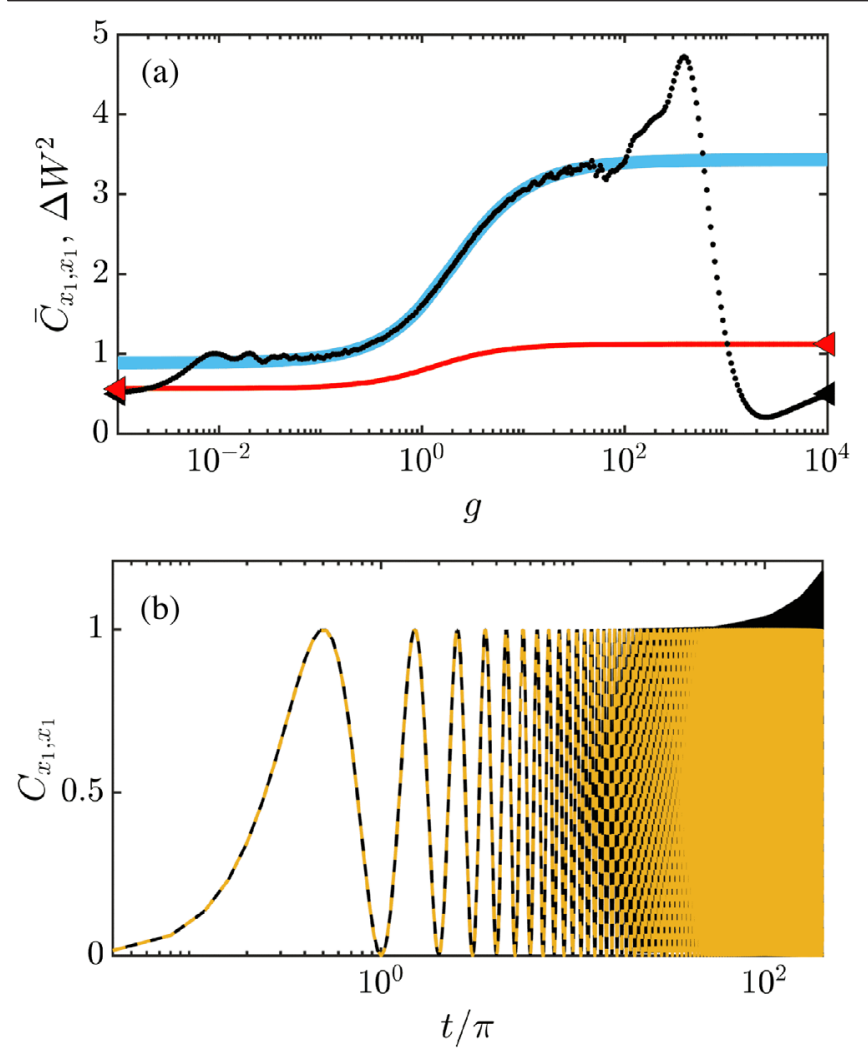

FIG. 2. (a) The full blue line is the infinite-time average of the squared commutator $\bar{C}_{x_{1}, x_{1}}$ as a function of $g$ for $\gamma=\frac{1}{2}$. Black dots show the time average of the same squared commutator in an interval $t \in[0,200 \pi]$ as a function of $g$. The variance of the work distribution function $\Delta W^{2}$ is given by the red solid line. The triangles correspond to the $g=0$ and $g=\infty$ values of $\bar{C}_{x x}$ (black) and $\Delta W^{2}$ (red). (b) $C_{x_{1}, x_{1}}(t)$ as a function of time for $g=0.002$ (black) and $g=0$ (yellow dashed) with $\gamma=\frac{1}{2}$.

[black dots in Fig. 2(a)]. For intermediate values of $g \in$ $[0.1,70]$ these results are indistinguishable from each other, but as the extremal interaction limits are approached the finite time-average and infinite time-average results diverge. For the $g=0$ limit the dynamics are shown in Fig. 2(b) and are simply given by $C_{\hat{x}_{1}, \hat{x}_{1}}(t)=\sin ^{2}(t)$ (yellow dashed line). It is interesting to compare them to the case of weak interactions, $g=0.002$, which possesses equivalent dynamics on short timescales (black solid line). In this case the interaction induced energy shift $\Delta_{j}$ is small and decreases as $\Delta_{j} \propto j^{-1 / 2}$ [52], such that the dynamics on short times can be approximated as $e^{-i\left(E_{j}^{0}+\Delta_{j}\right) t} \approx e^{-i\left(E_{j}^{0}\right) t}$ with $E_{j}^{0}$ being the single particle harmonic oscillator energies. However, at long times these energy shifts will affect the dynamics, leading to a change in the time average that is captured by Eq. (11). This discontinuity in the average information scrambling is therefore only observable in the long-time limit as the timescale required to observe the average scrambling diverges (similar for the $g \rightarrow \infty$ case).
In summary, we have shown that for harmonically trapped interacting atoms, which are a fundamental building block in many cold-atom experiments, the time average of the squared commutator $C_{A B}(t)$ for canonical operators is proportional to the work fluctuations. The operator scrambling in Hilbert space is therefore intimately linked to the work probability distribution, which is an experimentally accessible thermodynamic measure [21-23] of the nonequilibrium excitations induced by the quench. However, the timescale required to observe information scrambling is interaction dependent, being shorter the further the system is from the harmonic limits. In fact it diverges as the noninteracting and TG limits are approached, highlighting the importance of intermediate interactions to be able to observe information scrambling on short timescales. The relative lack of finite-size effects is curious and a further investigation of the moments of the work probability distribution as a function of $g$ and $N$ in a harmonic trap is an interesting line for future investigations. It would also be interesting to investigate other potentials which obey conditions (i)-(iii), but likely not condition (iv), in order to contrast and compare with the case of harmonic trapping.

This work was supported by the Okinawa Institute of Science and Technology Graduate University and utilized the computing resources of the Scientific Computing and Data Analysis section of the Research Support Division at OIST. In addition, M. M. was supported by the Japan Society for the Promotion through the JSPS fellowship (JSPS KAKENHI Grant No. 19J10852). T. F. acknowledges support under JSPS KAKENHI-21K13856.

*mathias-mikkelsen@phys.kindai.ac.jp

[1] A. Silva, Phys. Rev. Lett. 101, 120603 (2008).

[2] C. Karrasch and D. Schuricht, Phys. Rev. B 87, 195104 (2013).

[3] S. Campbell, Phys. Rev. B 94, 184403 (2016).

[4] M. Heyl, Rep. Prog. Phys. 81, 054001 (2018).

[5] T. Fogarty, A. Usui, Th. Busch, A. Silva, and J. Goold, New J. Phys. 19, 113018 (2017).

[6] N. Fläschner, D. Vogel, M. Tarnowski, B. S. Rem, D.-S. Lühmann, M. Heyl, J. C. Budich, L. Mathey, K. Sengstock, and C. Weitenberg, Nat. Phys. 14, 265 (2018).

[7] M. Mikkelsen, T. Fogarty, and T. Busch, New J. Phys. 20, 113011 (2018).

[8] A. del Campo, Phys. Rev. A 84, 012113 (2011).

[9] J. Goold, T. Fogarty, N. Lo Gullo, M. Paternostro, and Th. Busch, Phys. Rev. A 84, 063632 (2011).

[10] M. Cetina, M. Jag, R. S. Lous, J. T. M. Walraven, R. Grimm, R. S. Christensen, and G. M. Bruun, Phys. Rev. Lett. 115, 135302 (2015).

[11] M. M. Parish and J. Levinsen, Phys. Rev. B 94, 184303 (2016).

[12] M. Cetina, M. Jag, R. S. Lous, I. Fritsche, J. T. M. Walraven, R. Grimm, J. Levinsen, M. M. Parish, R. Schmidt, M. Knap, and E. Demler, Science 354, 96 (2016). 
[13] R. Schmidt, M. Knap, D. A. Ivanov, J.-S. You, M. Cetina, and E. Demler, Rep. Prog. Phys. 81, 024401 (2018).

[14] M. Rigol, V. Dunjko, V. Yurovsky, and M. Olshanii, Phys. Rev. Lett. 98, 050405 (2007).

[15] M. Rigol, V. Dunjko, and M. Olshanii, Nature (London) 452, 854 (2008).

[16] L. D’Alessio, Y. Kafri, A. Polkovnikov, and M. Rigol, Adv. Phys. 65, 239 (2016).

[17] L. Vidmar and M. Rigol, J. Stat. Mech. (2016) 064007 .

[18] M. A. García-March, T. Fogarty, S. Campbell, T. Busch, and M. Paternostro, New J. Phys. 18, 103035 (2016).

[19] A. Polkovnikov, Ann. Phys. (Amsterdam) 326, 486 (2011).

[20] M. Campisi, P. Hänggi, and P. Talkner, Rev. Mod. Phys. 83, 771 (2011).

[21] L. Fusco, S. Pigeon, T. J. G. Apollaro, A. Xuereb, L. Mazzola, M. Campisi, A. Ferraro, M. Paternostro, and G. De Chiara, Phys. Rev. X 4, 031029 (2014).

[22] T. B. Batalhão, A. M. Souza, L. Mazzola, R. Auccaise, R. S. Sarthour, I. S. Oliveira, J. Goold, G. De Chiara, M. Paternostro, and R. M. Serra, Phys. Rev. Lett. 113, 140601 (2014).

[23] F. Cerisola, Y. Margalit, S. Machluf, A. J. Roncaglia, J. P. Paz, and R. Folman, Nat. Commun. 8, 1241 (2017).

[24] T. Keller and T. Fogarty, Phys. Rev. A 94, 063620 (2016).

[25] M. Esposito, U. Harbola, and S. Mukamel, Rev. Mod. Phys. 81, 1665 (2009).

[26] J. D. Jaramillo, J. Deng, and J. Gong, Phys. Rev. E 96, 042119 (2017).

[27] D. Nigro, D. Rossini, and E. Vicari, J. Stat. Mech. (2019) 023104 .

[28] B. Swingle, Nat. Phys. 14, 988 (2018).

[29] R. J. Lewis-Swan, A. Safavi-Naini, A. M. Kaufman, and A. M. Rey, Nat. Rev. Phys. 1, 627 (2019).

[30] J. Maldacena, S. H. Shenkerband, and D. Stanford, J. High Energy Phys. 08 (2016) 106.

[31] M. Heyl, F. Pollmann, and B. Dóra, Phys. Rev. Lett. 121, 016801 (2018).

[32] C.-J. Lin and O. I. Motrunich, Phys. Rev. B 97, 144304 (2018).

[33] C. B. Dağ, K. Sun, and L.-M. Duan, Phys. Rev. Lett. 123, 140602 (2019).

[34] Martin Gärttner, J. G. Bohnet, A. Safavi-Naini, M. L. Wall, J. J. Bollinger, and A. M. Rey, Nat. Phys. 13, 781 (2017).

[35] M. McGinley, A. Nunnenkamp, and J. Knolle, Phys. Rev. Lett. 122, 020603 (2019).

[36] M. Campisi and J. Goold, Phys. Rev. E 95, 062127 (2017).

[37] R. J. Lewis-Swan, A. Safavi-Naini, J. J. Bollinger, and A. M. Rey, Nat. Commun. 10, 1581 (2019).

[38] R. Hamazaki, K. Fujimoto, and M. Ueda, arXiv:1807.02360.

[39] N. Yunger Halpern, Phys. Rev. A 95, 012120 (2017).
[40] A. Chenu, J. Molina-Vilaplana, and A. del Campo, Quantum 3, 127 (2019).

[41] B. Yan, L. Cincio, and W. H. Zurek, Phys. Rev. Lett. 124, 160603 (2020).

[42] G. Zürn, F. Serwane, T. Lompe, A. N. Wenz, M. G. Ries, J. E. Bohn, and S. Jochim, Phys. Rev. Lett. 108, 075303 (2012).

[43] M. Endres et al., Science 354, 1024 (2016).

[44] A. N. Wenz et al., Science 342, 457 (2013).

[45] A. Minguzzi and D. M. Gangardt, Phys. Rev. Lett. 94, 240404 (2005).

[46] Y. Y. Atas, D. M. Gangardt, I. Bouchoule, and K. V. Kheruntsyan, Phys. Rev. A 95, 043622 (2017).

[47] See Supplemental Material at http://link.aps.org/ supplemental/10.1103/PhysRevLett.128.070605. This contains a derivation of the squared commutator in the noninteracting and $\mathrm{TG}$ case, the derivation of the squared commutator in terms of $\mathrm{CM}$ and relative parts in the interacting case and a proof of condition (iii) for the relative part of the interacting Hamiltonian with respect to the Jacobi coordinates. Details about the analytic description of the $N=2$ correlation functions and numerical comparisons between different canonical operators is also contained. Supplemental Material includes Refs. [48-50].

[48] M. Girardeau, J. Math. Phys. (N.Y.) 1, 516 (1960).

[49] S. Waldensrøm and K. Razi Naqvi, Chem. Phys. Lett. 85, 581 (1982).

[50] D. Baye, Phys. Status Solidi B 243, 1095 (2006).

[51] T. Sowiński and M. A. García-March, Rep. Prog. Phys. 82, 104401 (2019).

[52] T. Busch, B.-G. Englert, K. Rzazewski, and M. Wilkens, Found. Phys. 28, 549 (1998).

[53] L. Budewig, S. I. Mistakidis, and P. Schmelcher, Mol. Phys. 117, 2043 (2019).

[54] M. A. García-March and T. Busch, Phys. Rev. A 87, 063633 (2013).

[55] E. J. Lindgren, J. Rotureau, C. Forssén, A. G. Volosniev, and N. T. Zinner, New J. Phys. 16, 063003 (2014).

[56] M. Płodzień, D. Wiater, A. Chrostowski, and T. Sowiński, arXiv:1803.08387.

[57] M. Srednicki, J. Phys. A 32, 1163 (1999).

[58] N. L. Harshman, Phys. Rev. A 86, 052122 (2012).

[59] N. L. Harshman, Few-Body Syst. 57, 11 (2016).

[60] N. L. Harshman, Few-Body Syst. 57, 45 (2016).

[61] A. S. Dehkharghani, A. G. Volosniev, and N. T. Zinner, J. Phys. B 49, 085301 (2016).

[62] R. E. Barfknecht, A. S. Dehkharghani, A. Foerster, and N. T. Zinner, J. Phys. B 49, 135301 (2016).

[63] K. Hashimoto, K. Murata, and R. Yoshii, J. High Energy Phys. 10 (2017) 138. 